Monatsschrift für Geburtshülfe u. Gynäkologie 1908;28:I-V

\title{
Contents, Vol. 28, 1908
}

\section{Inhalts - Verzeichnis}

Original- Arbeiten.

Alexandroff, Theodor, Ein Fall von Hyperemesis gravi-

darum, kompliziert mit einer Korsakoffschen Psychose 542

Bauer, Adolf, Spitze Condylome von besonderer Grösse. 297

Becker, Georg, Die Achsendrehung des Dickdarms in Be-

ziehung zu Schwangerschaft und Gebuit 155

Birnbaum, Richard, und Gustav Thalheim, Unter-

suchungen tiber die chemische Zusammensetzung der

Myome und der Uterusmuskulatur 509

Bokofzer, S., Hat der Druck der Rumpílast Einfluss auf

die Formbildung des Beckens ? 135

Bondi, Josef, Zur Anatomie der Cysten der kleinen Scham-

lippe 648

Frankenstein, Kurt, Ueber klinische Erfahrungen mit

der Vaporisation 396

Friedjung, Josef K., Beitrag zur Kenntnis der Spät-

laktation 639

Cans, H., Typhusvereiterung des Ovarialtumors

$\mathrm{G}$ o e $\mathrm{n} \mathrm{n}$ er , A1 f r e d, Ueber Sondenaufrichtung und Intrauterin-

stifte 78

Go th, Laj os , Fibroma multiplex cutis bei einer Schwangeren 423 Hellendall, H., Zur

obligatorischen Crédéisierung der Neu-

geborenen 283

Henrich, Otto, Ueber einen Fall von Uterus- und Tuben-

tuberkulose in Verbindung mit Myom 383

Himmelheber, Kurt, DieErgebnisse der Blutuntersuchung

in prognostischer Hinsicht beim Wochenbettfieber . . 30Ü

Hofbauer, J., Larynx und Schwangerschaft. Klinische

Studie 45

- IV

Hofbauer, J., Histologische Besonderheiten von Vagina

und Blase während der Gravidität 131

Jarzef f, A., Zur Frage über die Bauchhöhlenschwangerschaft 144 J as c like, Rud. Th., Die Bedeutung des Selbststillens im Kampfe gegendieSäuglingssterblichkeit; bestehendeEinrichtungen und Vorschläge zur Förderung derselben 172, 257 Martin , A. , Offener Brief an die Herren Professoren Krönig

undSellheirn in der Frage der Placenta praevia-Therapie . 638a Mathes, P., Ueber Aetiologie und Therapie der Dysmenor- 
rhoe 73

Rosenfeld, Wilhelm, Ueber Kraurosis vulvae

60

Rosinski, B., Ueber missed abortion 108

Schaffer, Josef, Ueber Bau und Funktion des Eileiter-epithels beim Menschen und bei

Säugetieren. (Hierzu

Tafel I-II) 526, 666

Schmidlechner, Carl, Primäre und Dauerresultate der Ovariotomien bei anatomisch malignen und zweifel-

haften Geschwülsten 1

Schubert, Gotthard, Herzlose Missgeburten 312

Schwab, Max, Ein Beitrag zum hysterischen Fieber . . 414

Stern, Rob., Ueber den Vorfall der Nachgeburt bei nor-

raalem Sitz. (Nachtrag zu der Arbeit unter gleichem

Titel in Bd. XXVII, S. 685.) 200

Zwei Gynäkologen-Versammlungen in den Vereinigten Staaten. (American gynecological

Society, Philadelphia, 26. bis 28. Mai, und Geburtshülflich-Gynäkologische Sektion der

American Medical Association, 2.-4. Juni

1908.) Von A. Martin in Berlin 431

Köln 1908. Von A. Martin in Berlin; 555

Die neuen Frauenkliniken in Wien. Von A. Martin in Berlin 597

Sammelreferate.

Die geburtshülfliche und gynäkologische Literatur in Nord-

Ainerika 1907, III. und IV. Quartal. Von Arthur

Stein in New-York 201, 325

Bericht über die französischen geburtshülflich-gynäkologi-

schen Arbeiten des Jahres 1908. Von Cyrille Jeannin

in Paris 689

Die geburtshülfliche und gynäkologische Literatur in Holland

1907, I. Halbjahr. Von M. M. de Monchy in Haag 696

- v-

Polnische geburtshülfliche und gynäkologischeLiteratur 1907.

Von Hugo Ehrlich in Przemysl 708

Sammelbericht über die im Jahre 1907 in Rumänien ver-

öffentlichten Arbeiten gynäkologischen und geburts-

hülflichen Inhalts. Von E. Toff in Braila 84

80. Versammlung deutscher Naturforscher und

Aerzte in Köln a. Rh. vom 20.-25. Sept. 1908.

Bericht von Kurt Frankenstein in Köln 556

33. Jahresversammlung der amerikanischen gynäko

logischen Gesellschaftzu Philadelphia, 26., 27.und

28. Mai 1908. Bericht von Arthur Stein in New-

York 475

Personalien und Tagesnaehrichten $\quad$ 213, 434, 599, 717

Vereins- und Literaturbeilage.

Original-Sitzungsberichte aus geburtshülflich-gynäkologischen

Gesellschaften: 
Gynäkologische Gesellschaft zu Breslau 92, 338, 435 Kölner Gesellschaft für Geburtshülfe und Gynäkologie 355,

Mittelrheinische Gesellschaft für Geburtshülfe und Gynäkologie 351

Münchener gynäkologische Gesellschaft $\quad 600,718$

Niederrheinisch-westfälische Gesellschaft für Gynäkologie und Geburtshülfe 449

Ost- und westpreussische Gesellschaft für Gynäkologie . . 219

Referate aus geburtshülflich-gynäkologischen Gesellschaften

113, 233, 367, 490, 625, 735

Geburtshüflich-gynäkologische Mitteilungen aus anderen Ge

sellschaften uud Vereinen . . 118, 243, 371, 490, 627, 738

Buchanzeigen 123, 247, 375, 493, 631, 739

Literatur-Verzeichnis 124, 249, 376, 503, 633, 742

Sachregister $\quad 747$

Namenregister $\quad 758$ 